\title{
Optically Levitated Nanodumbbell Torsion Balance and GHz Nanomechanical Rotor
}

\author{
Jonghoon Ahn, ${ }^{1}$ Zhujing Xu, ${ }^{2}$ Jaehoon Bang, ${ }^{1}$ Yu-Hao Deng, ${ }^{3}$ Thai M. Hoang,,${ }^{2, *}$ Qinkai Han, ${ }^{4, \dagger}$ \\ Ren-Min Ma, ${ }^{3,5, \$}$ and Tongcang $\mathrm{Li}^{1,2,6,7, \S}$ \\ ${ }^{1}$ School of Electrical and Computer Engineering, Purdue University, West Lafayette, Indiana 47907, USA \\ ${ }^{2}$ Department of Physics and Astronomy, Purdue University, West Lafayette, Indiana 47907, USA \\ ${ }^{3}$ State Key Lab for Mesoscopic Physics and School of Physics, Peking University, Beijing 100871, China \\ ${ }^{4}$ The State Key Laboratory of Tribology, Tsinghua University, Beijing 100084, China \\ ${ }^{5}$ Collaborative Innovation Center of Quantum Matter, Beijing 100871, China \\ ${ }^{6}$ Purdue Quantum Center, Purdue University, West Lafayette, Indiana 47907, USA \\ ${ }^{7}$ Birck Nanotechnology Center, Purdue University, West Lafayette, Indiana 47907, USA
}

(Received 18 April 2018; published 20 July 2018)

\begin{abstract}
Levitated optomechanics has great potential in precision measurements, thermodynamics, macroscopic quantum mechanics, and quantum sensing. Here we synthesize and optically levitate silica nanodumbbells in high vacuum. With a linearly polarized laser, we observe the torsional vibration of an optically levitated nanodumbbell. This levitated nanodumbbell torsion balance is a novel analog of the Cavendish torsion balance, and provides rare opportunities to observe the Casimir torque and probe the quantum nature of gravity as proposed recently. With a circularly polarized laser, we drive a 170-nm-diameter nanodumbbell to rotate beyond $1 \mathrm{GHz}$, which is the fastest nanomechanical rotor realized to date. Smaller silica nanodumbbells can sustain higher rotation frequencies. Such ultrafast rotation may be used to study material properties and probe vacuum friction.
\end{abstract}

DOI: $10.1103 /$ PhysRevLett.121.033603

Levitated optomechanical systems provide a powerful platform for precision measurements with great isolation from the thermal environment [1-6]. Optically levitated nano- and microspheres have been used to demonstrate force sensing at the level of $10^{-21} \mathrm{~N}$ [7] and to search for interactions associated with dark energy [8]. Optically trapped nanoparticles can also be driven to rotate at high speed. Previously, a rotation frequency of about $10 \mathrm{MHz}$ had been achieved [9-11]. It is desirable to increase the rotation frequency further for studying material properties under extreme conditions [12,13] and probing vacuum friction [14-16].

Recently, a novel ultrasensitive torsion balance with an optically levitated nonspherical nanoparticle was proposed [17], utilizing the coupling between the spin angular momentum of photons and the mechanical motion of the nanoparticle [4,17-20]. Torsion balances have enabled many breakthroughs in the history of modern physics [21-24]. For example, the Cavendish torsion balance [Fig. 1(a)] determined the gravitational constant and the density of the Earth [21]. An optically levitated nanoscale torsion balance can provide a rare opportunity to detect the Casimir torque [25-28], and test the quantum nature of gravity [29-31]. An essential step towards these goals is to optically trap a well-defined nonspherical nanoparticle in high vacuum. However, optically trapped nonspherical nanoparticles such as nanodiamonds and silicon nanorods in former experiments were lost at about 1 torr due to laser heating [10,17,32-34]. Additionally, several years ago, levitated nanodumbbells were theoretically proposed to study many-body phase transitions [19]. To the best of our knowledge, however, there has been no report on optical levitation of a nanodumbbell in vacuum prior to this work.

In this Letter, we synthesize silica nanodumbbells with two different methods and optically trap them in high vacuum. With a linearly polarized laser [Fig. 1(b)], we observe the torsional vibration of a levitated nanodumbbell in high vacuum, which is an important step towards probing the Casimir torque [25-28] and the quantum nature of gravity [29-31]. With a circularly polarized laser, we drive the nanodumbbell to rotate beyond $1 \mathrm{GHz}$, which is the highest mechanical rotation frequency reported to date.

In a linearly polarized optical tweezer, the long axis of a nanodumbbell will tend to align with the polarization direction of the trapping laser [Fig. 1(b)]. This is because the polarizability of the nanodumbbell along its long axis is larger than the polarizability perpendicular to its long axis. If the nanodumbbell is not aligned with the polarization direction of the optical tweezer, it will twist the polarization of the optical tweezer [Fig. 1(b)], as an analog of twisting the torsion wire by the lead spheres in the original Cavendish torsion balance [Fig. 1(a)]. If the optical tweezer is circularly polarized, the nanodumbbell will be driven to rotate at high speed. The torsional vibration or rotation of the nanodumbbell can be detected by monitoring the change of the polarization of the trapping laser [Fig. 1(c)]. 
(a)
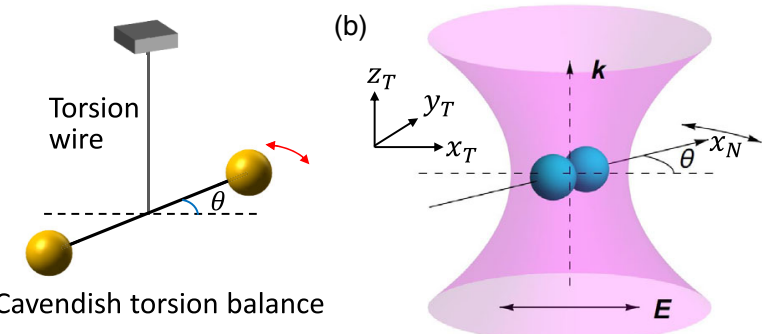

(c)

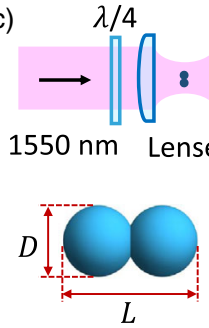

(b)

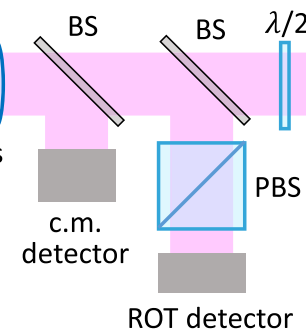

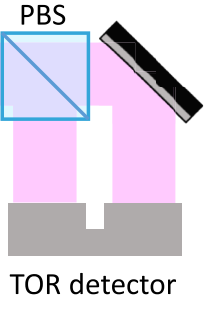

FIG. 1. (a) A simplified diagram of the original Cavendish torsion balance that has two lead spheres suspended by a copper silvered torsion wire. (b) A nanodumbbell levitated by a linearly polarized optical tweezer in vacuum. The linearly polarized optical tweezer provides the restoring torque that confines the orientation of the nanodumbbell. $x_{T}, y_{T}, z_{T}$ are Cartesian coordinates of the trapping laser. $x_{T}$ is parallel to the electric field $\mathbf{E}$ of the incoming linearly polarized laser, and $z_{T}$ is parallel to the wave vector $\mathbf{k}$ of the laser. $x_{N}$ is parallel to the long axis of the nanodumbbell. The angle between $x_{T}$ and $x_{N}$ is $\theta$. (c) $\mathrm{A}$ simplified diagram for detecting the center-of-mass (c.m.) motion, the torsional (TOR) vibration, and the rotation (ROT) of a levitated nanodumbbell. The nanodumbbell is trapped at the focus of the lenses. The laser beam is initially linearly polarized. A quarter-wave $(\lambda / 4)$ plate is used to control its polarization. BS: non-polarizing beam splitter; PBS: polarizing beam splitter; $\lambda / 2$ : half-wave plate. Inset: A nanodumbbell with diameter $D$ and length $L$ created by attaching two identical nanospheres.

We have synthesized pure silica nanodumbbells using chemical and physical methods. The interpenetration of the two particles in a nanodumbbell is tunable. To synthesize nanodumbbells with diameter $D$ and length $L$ [inset of Fig. 1(c)], we first synthesize silica cores with a diameter of $d=L-D$ by adding tetraethyl orthosilicate (TEOS) to a mixture of ammonia, pure water, and ethanol under stirring $[35,36]$. Then acetone is added into the solution to induce aggregation. Next, a small amount of TEOS is added under stirring to grow the silica shells [35]. This chemical method can synthesize a large quantity of silica nanodumbbells with a tunable aspect ratio [35], but is demanding. So we also develop a physical method to assemble nanodumbbells. In this method, we first prepare a colloidal suspension of silica nanospheres in water. We then generate water microdroplets in air with an ultrasonic nebulizer [33]. By controlling the concentration of silica nanospheres, a fraction of water microdroplets ( $\sim 5 \mu \mathrm{m}$ in diameter) contain 2 silica nanospheres in them. Two nanospheres in the same microdroplet will form a nanodumbbell as the water

(a)
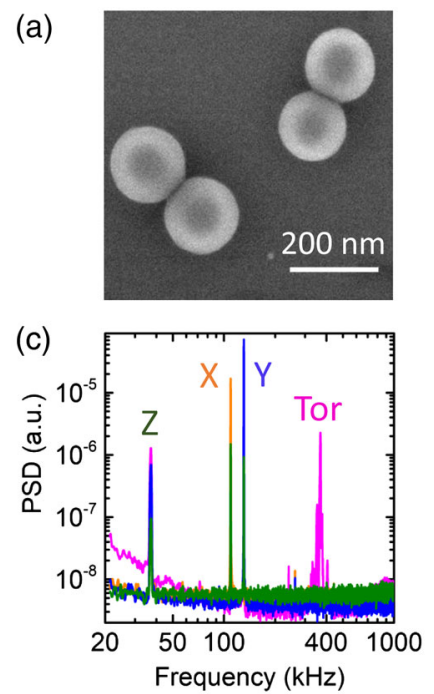

(b)
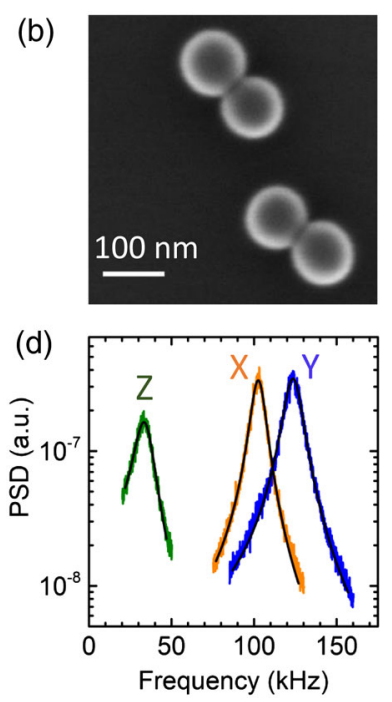

FIG. 2. (a),(b): SEM images of silica nanodumbbells with two different sizes. The scale bar is $200 \mathrm{~nm}$ in (a) and $100 \mathrm{~nm}$ in (b). (c) Measured power spectrum densities (PSD) of the torsional vibration (labeled "Tor") and the translational vibrations (labeled " $X$," " $Y$," " $Z$ ") of a 170-nm-diameter nanodumbbell optically levitated at $5 \times 10^{-4}$ torr. (d) Measured PSD of the translational vibrations of the nanodumbbell levitated at 10 torr. The black curves are Lorentz fits.

evaporates. Figures 2(a) and 2(b) show SEM images of our nanodumbbells in two different sizes. Their aspect ratio is between 1.9 and 2.

To optically levitate a silica nanodumbbell in vacuum, a $500 \mathrm{~mW}, 1550 \mathrm{~nm}$ laser is tightly focused with an NA = 0.85 objective lens in a vacuum chamber. The laser is initially linearly polarized, and its polarization can be controlled with a quarter wave plate [Fig. 1(c)]. Silica nanodumbbells are delivered into the optical trap at atmospheric pressure with an ultrasonic nebulizer [33]. Once a nanoparticle is trapped, we evacuate the vacuum chamber to below 0.01 torr, and then increase the pressure back to desired levels for measurements. This procedure removes extra nanoparticles in the chamber. To monitor the trapping process, a $532 \mathrm{~nm}$ laser is applied on the nanoparticle and the scattered light is viewed using a camera. We verify the trapped nanoparticle is a nanodumbbell by checking the ratios of damping coefficients for translational motions along different directions. The motion of the levitated nanoparticle changes the direction and polarization of the laser beam, which allows us to monitor the motion of a nanodumbbell with the same $1550 \mathrm{~nm}$ trapping laser [Fig. 1(c)] [17]. Figure 2(c) shows the power spectrum density (PSD) of both torsional (TOR) vibration and centerof-mass (c.m.) vibration of a levitated 170-nm-diameter nanodumbbell in vacuum at $5 \times 10^{-4}$ torr. This is an important step towards quantum ground state cooling of the torsional vibration and testing recent theoretical proposals [25,29-31]. 
A levitated nanodumbbell will have anisotropic damping rates for translational motions in air if its orientation is fixed. We use the direct simulation Monte Carlo (DSMC) method to obtain the damping force and damping torque of a nanodumbbell in the free molecular flow regime [36-39]. In the simulation, molecules with uniform spatial distribution are launched from a spherical surface enclosing the nanodumbbell [36]. The speeds of these molecules satisfy a shifted Maxwell distribution to include the effect of the motion of the nanodumbbell [39]. Figure 3(a) shows the calculated ratio of damping rates of a nanodumbbell moving along $\left(\Gamma_{x}\right)$ and perpendicular $\left(\Gamma_{y}\right)$ to its axial direction. The calculated ratio is $\Gamma_{y} / \Gamma_{x}=\Gamma_{z} / \Gamma_{x}=1.276$ when $L / D=1.9$, and $\Gamma_{y} / \Gamma_{x}=\Gamma_{z} / \Gamma_{x}=1.258$ when $L / D=2$. The measured ratios for the data of a $170 \mathrm{~nm}$ nanodumbbell shown in Fig. 2(d) are $\Gamma_{y} / \Gamma_{x}=1.25 \pm 0.01$ and $\Gamma_{z} / \Gamma_{x}=1.27 \pm 0.02$, which agree excellently with our theoretical predictions. The DSMC method is also utilized to obtain the drag torque $T_{z}$ on a nanodumbbell rotating at speed $\Omega$. We then calculate the ratio $T_{z} / T_{\text {sphere, }}$, where $T_{\text {sphere }}=\pi \mu D^{4} \Omega /\left(11.976 \lambda_{M}\right)$ is the drag torque on a single sphere with diameter $D$ rotating at the same speed $\Omega$ [39]. Here $\mu$ is the viscosity of air, and $\lambda_{M}$ is the mean free path of air molecules which is inversely proportional to air
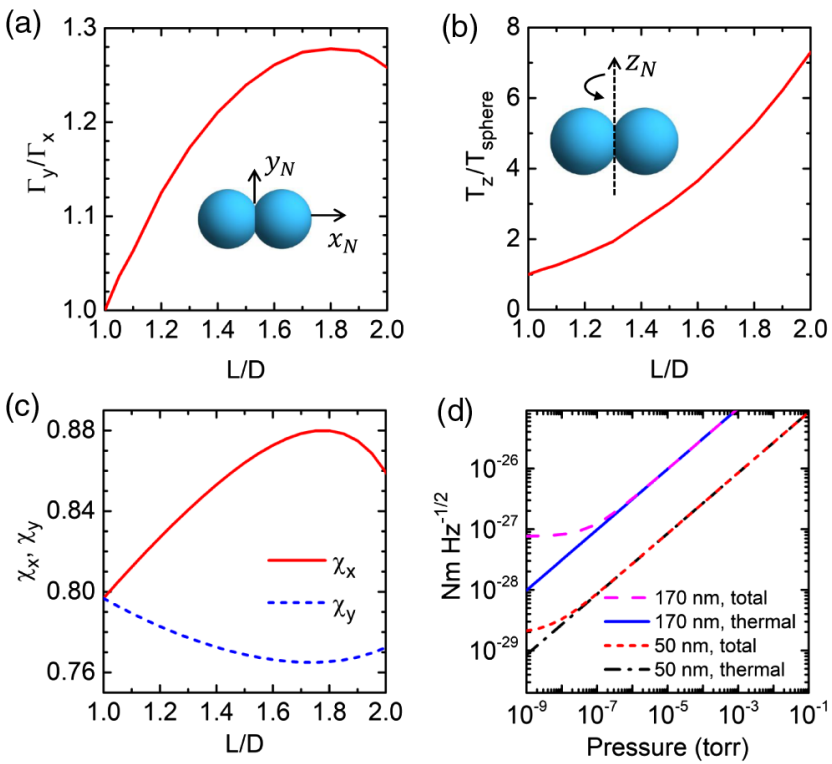

FIG. 3. (a) The ratio of air damping coefficients for translational motions perpendicular or parallel to its long axis $\left(x_{N}\right.$ axis) as a function of the aspect ratio $(L / D)$ of a nanodumbbell. (b) Calculated normalized drag torque of the rotation of a levitated nanodumbbell around $z_{N}$ axis as a function of the aspect ratio. (c) Effective susceptibilities of a silica nanodumbbell parallel $\left(\chi_{x}\right)$ or perpendicular $\left(\chi_{y}\right)$ to its long axis. (d) Calculated torque detection sensitivity of a levitated nanodumbbell with $D=$ $170 \mathrm{~nm}$ or $D=50 \mathrm{~nm}$ as a function of air pressure. We assume $L / D=1.9$ in the calculations. The optical tweezer is assumed to be a focused $500 \mathrm{~mW}, 1550 \mathrm{~nm}$ laser with a waist of $820 \mathrm{~nm}$. pressure $p_{\text {air }}$. The calculated results are shown in Fig. 3(b). The damping rate of the rotation or torsional vibration around the $z$ axis is $\Gamma_{\theta}=T_{z} /\left(I_{z} \Omega\right)$, where $I_{z}$ is its moment of inertia.

When the size of a silica nanodumbbell is much smaller than the wavelength of the trapping laser, the dipole approximation can be applied. The complex amplitude of the induced dipole of the nanodumbbell is $\mathbf{p}=\alpha_{x} E_{x} \hat{x}_{N}+\alpha_{y} E_{y} \hat{y}_{N}+\alpha_{z} E_{z} \hat{z}_{N}$, where the complex amplitude of the electric field of the laser beam $\mathbf{E}$ is decomposed into components along the principle axes of the nanodumbbell. $\hat{x}_{N}$ is in the direction along the long axis of the nanodumbbell. The components of the optical force $F_{j}$ and the optical torque $M_{j}$ acting on the nanodumbbell can be expressed as [40] $F_{j}=\frac{1}{2} \operatorname{Re}\left\{\mathbf{p}^{*} \cdot \partial_{j} \mathbf{E}\right\}$ and $M_{j}=\frac{1}{2} \operatorname{Re}\left\{\mathbf{p}^{*} \times \mathbf{E}\right\}_{j}$. The quasistatic polarizability $\alpha_{j}^{0}$ $(j=x, y, z)$ of a nanodumbbell can be calculated assuming the electric field is static [41]. Figure 3(c) shows the effective susceptibilities $\chi_{x}=\alpha_{x}^{0} /\left(\epsilon_{0} V\right), \chi_{y}=\alpha_{y}^{0} /\left(\epsilon_{0} V\right)$ of the nanodumbbell as a function of the aspect ratio. Here $\epsilon_{0}$ is the permittivity of vacuum and $V$ is the volume of the nanodumbbell. $\left(\chi_{x}-\chi_{y}\right) / \chi_{y}=0.14$ when $L / D=1.9$. The dipole approximation can be improved by including the effects of radiation reaction due to the oscillation of the electric field in a laser beam. Then the polarizability is $[40,42,43] \quad \alpha_{j}=\alpha_{j}^{0} /\left[1-i k_{0}^{3} \alpha_{j}^{0} /\left(6 \pi \epsilon_{0}\right)\right]$, where $k_{0}$ is the wave number. The real part of the polarizability $\operatorname{Re}\left[\alpha_{j}\right] \approx \alpha_{j}^{0}$ is responsible for optical confinement and alignment, while the imaginary part $\operatorname{Im}\left[\alpha_{j}\right]$ is important for optically rotating a nanodumbbell.

With calculated damping rates and polarizabilities, we can calculate the torque detection sensitivity of a levitated nanodumbbell. The minimum torque that it can measure limited by thermal noise is [44] $M_{\mathrm{th}}=\sqrt{4 k_{B} T_{\mathrm{env}} I_{z} \Gamma_{\theta} / \Delta t}$, where $T_{\text {env }}$ is the environmental temperature. In ultrahigh vacuum, the thermal noise from the residual air molecules becomes negligible and the minimum torque it can detect will be limited by the shot noise of the laser beam [25]: $M_{\mathrm{rad}}=\left(\chi_{x}-\chi_{y}\right) k_{0}^{2} V \hbar \sqrt{J_{p} /(3 \pi \Delta t)}$. Here, $J_{p}=I_{\text {laser }} /\left(\hbar \omega_{L}\right)$ is the photon flux. $I_{\text {laser }}$ is the laser intensity, and $\omega_{L}$ is the angular frequency of the laser. As shown in Fig. 3(d), at pressures below $10^{-8}$ torr, a nanodumbbell with $D=170 \mathrm{~nm}$ and $D=50 \mathrm{~nm}$ trapped in a $500 \mathrm{~mW}$ laser will have a torque detection sensitivity of about $10^{-27} \mathrm{~N} \mathrm{~m} / \sqrt{\mathrm{Hz}}$ and $10^{-29} \mathrm{~N} \mathrm{~m} / \sqrt{\mathrm{Hz}}$, respectively. Remarkably, a levitated nanodumbbell at $10^{-4}$ torr is already much more sensitive than the current state-ofthe-art nanofabricated torsion balance, which has achieved a torque sensitivity of $10^{-22} \mathrm{~N} \mathrm{~m} / \sqrt{\mathrm{Hz}}$ at room temperature, and $10^{-24} \mathrm{Nm} / \sqrt{\mathrm{Hz}}$ at $25 \mathrm{mK}$ in a dilution refrigerator [45]. The original Cavendish experiment measured a torque of about $10^{-7} \mathrm{Nm}$ caused by gravitational attraction [21]. 
(a)

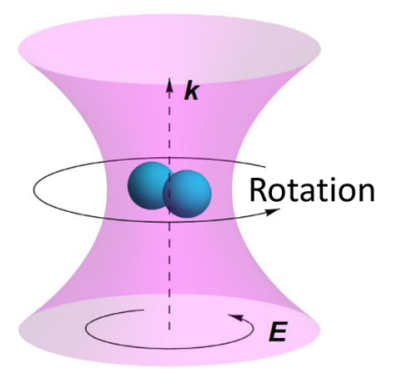

(d)

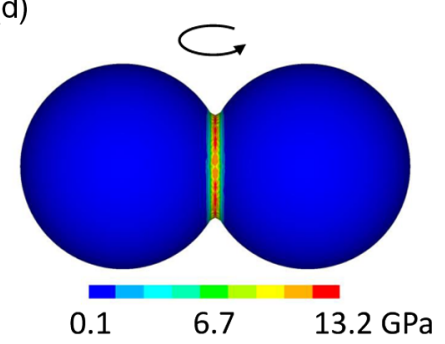

(b)

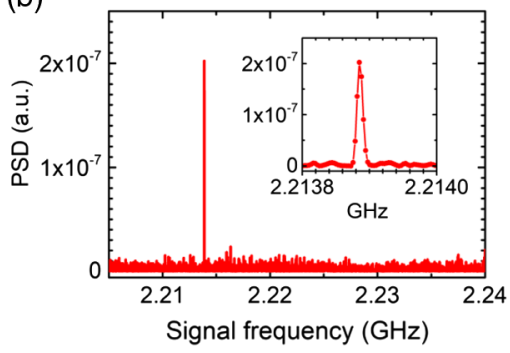

(e)

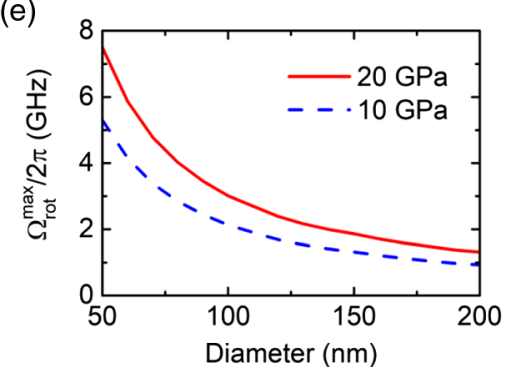

(c)

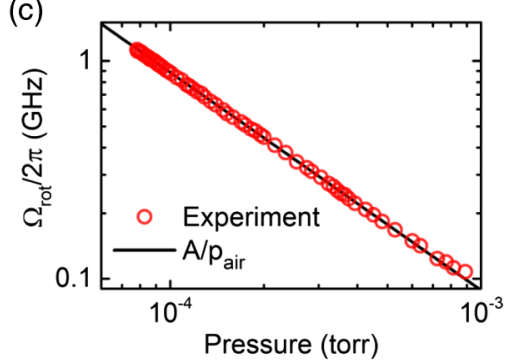

(f)

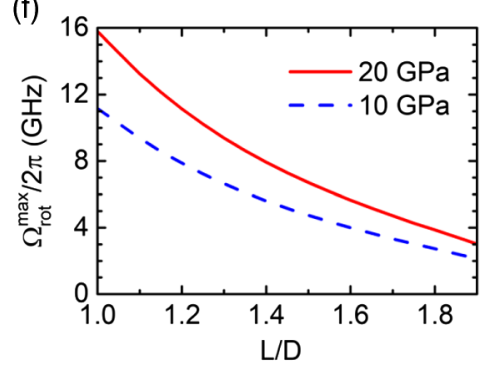

FIG. 4. (a) A nanodumbbell levitated by a circularly polarized laser will rotate. (b) Measured PSD of the rotation signal of a nanodumbbell. It has a sharp peak near $2.2 \mathrm{GHz}$. (c) Measured rotation frequency of the nanodumbbell as a function of pressure. (d) Calculated stress distribution of a nanodumbbell $(D=170 \mathrm{~nm}, L / D=1.9$ ) rotating at $1.2 \mathrm{GHz}$. (e) Calculated ultimate rotation frequency as a function of the diameter of the nanodumbbell when $L / D=1.9$. (f) Calculated ultimate rotation frequency as a function of the aspect ratio of the nanodumbbell when $D=100 \mathrm{~nm}$. The ultimate tensile strength is assumed to be $20 \mathrm{GPa}$ (red solid line) or $10 \mathrm{GPa}$ (blue dashed line).

While a nanodumbbell levitated by a linearly polarized optical tweezer can be an ultrasensitive nanoscale torsion balance, it will become an ultrafast nanomechanical rotor in a circularly polarized optical tweezer [Fig. 4(a)]. The frequency of the detected signal will be twice the rotation frequency of the nanodumbbell due to the symmetry of its shape. Figure 4(b) shows a PSD of the rotation of a 170 -nm-diameter nanodumbbell at $7.9 \times 10^{-5}$ torr. The detected signal has a sharp peak near $2.2 \mathrm{GHz}$. This shows the nanodumbbell rotates at $1.1 \mathrm{GHz}$, which is much faster than that from former experiments [9-11]. Remarkably, the rotation is also very stable. The measured linewidth of the signal is less than $20 \mathrm{kHz}$, limited by the resolution of our spectrum analyzer, which leads to a rotating quality factor of larger than $10^{5}$. The steady-state rotation speed is determined by the balance between the optical torque $M_{z}$ exerted on the nanodumbbell and the drag torque $T_{z}$ from air molecules. Since drag torque is proportional to the air pressure, the steady state rotation speed $\Omega_{\text {rot }}$ is inversely proportional to the air pressure $p_{\text {air }}$. Fig. 4(c) shows the rotation speed as a function of the air pressure. The experimental data agree with $A / p_{\text {air }}$, where $A$ is a fitting parameter. As the air pressure decreases, the rotation speed increases. The maximum rotation frequency that we can measure is limited by the bandwidth of our photodetector.

As the rotation speed increases, eventually the nanodumbbell will fall apart due to centrifugal force. The ultimate rotating frequency is determined by the ultimate tensile strength (UTS) of the material. Thus this experiment provides an opportunity to study material properties under extreme conditions. The stress distribution of a nanodumbbell rotating at high speed can be calculated by the finite element method. Figure 4(d) shows the stress distribution of a $D=170 \mathrm{~nm}, L / D=1.9$ silica nanodumbbell rotating at $1.2 \mathrm{GHz}$. We assume the contacting point has a curvature radius of $5 \mathrm{~nm}$. Remarkably, the maximum stress of the nanodumbbell under these conditions is about $13 \mathrm{GPa}$, which is 2 orders larger than the UTS of a bulk glass. This shows that our silica nanodumbbells are as strong as stateof-the-art silica nanowires [46]. The finite element method can be used to calculate the ultimate rotating frequency of silica nanodumbbells with different diameters and aspect ratios. The range of ultimate rotating frequency is calculated by setting the UTS to be in the range of 10-20 GPa, which agrees with our observation and the results for silica nanowires [46]. Figure 4(e) shows the calculated ultimate frequency is in the range of $1.1-1.6 \mathrm{GHz}$ for $D=170 \mathrm{~nm}, L / D=1.9$ nanodumbbells. The ultimate frequency increases as the size of the nanodumbbell decreases. Figure 4(f) shows that for a given diameter $D=100 \mathrm{~nm}$, the ultimate rotation frequency increases when the aspect ratio decreases. Thus nanodumbbells with smaller diameters and smaller aspect ratios can sustain rotation frequencies beyond $10 \mathrm{GHz}$.

In conclusion, we have synthesized and optically trapped nanodumbbells in high vacuum. When the laser is linearly polarized, the torsional vibration of the levitated nanodumbbell is observed, an important step towards detecting the Casimir torque [25] and developing a quantum Cavendish torsion balance for studying the quantum nature 
of gravity [29-31]. With a circularly polarized laser, 170nm-diameter nanodumbbells were driven to rotate beyond $1 \mathrm{GHz}$, which is the highest reported rotation frequency for a nanoparticle. Such high speed rotation may be used to study material properties and vacuum friction [12-16]. Based on Refs. [14,16], a 100-nm-diameter $\mathrm{SiC}$ nanosphere rotating at $1 \mathrm{GHz}$ near a $\mathrm{SiC}$ surface at $1000 \mathrm{~K}$ with a separation of $150 \mathrm{~nm}$ will experience a vacuum friction torque on the order of $10^{-28} \mathrm{~N} \mathrm{~m}$, which seems detectable at $10^{-8}$ torr.

We thank helpful discussions with F. Robicheaux and Z. Yin. T. L. is supported by NSF under Grant No. PHY1555035 and Office of Naval Research under Grant No. N00014-18-1-2371. R.-M. M. is supported by National Natural Science Foundation of China (Grants No. 11574012, No. 11774014, No. 61521004) and the "Youth 1000 Talent Plan." Q.H. is supported by the National Science Foundation of China under Grant No. 11472147, and the State Key Laboratory of Tribology under Grant No. SKLT2015B12.

Note added.- -Recently, we became aware of a related work on fast rotation of a nanoparticle [47].

*Present address: Sandia National Laboratories, Albuquerque, NM 87123, USA.

†hanqinkai@mail.tsinghua.edu.cn

†renminma@pku.edu.cn

$\S_{\text {tcli@purdue.edu }}$

[1] Z.-Q. Yin, A. A. Geraci, and T. Li, Int. J. Mod. Phys. B 27, 1330018 (2013).

[2] A. Ashkin and J. M. Dziedzic, Appl. Phys. Lett. 28, 333 (1976).

[3] T. Li, S. Kheifets, and M. G. Raizen, Nat. Phys. 7, 527 (2011).

[4] O. Romero-Isart, M. L. Juan, R. Quidant, and J. I. Cirac, New J. Phys. 12, 033015 (2010).

[5] D. E. Chang, C. A. Regal, S. B. Papp, D. J. Wilson, J. Ye, O. Painter, H. J. Kimble, and P. Zoller, Proc. Natl. Acad. Sci. U.S.A. 107, 1005 (2010).

[6] M. Frimmer, K. Luszcz, S. Ferreiro, V. Jain, E. Hebestreit, and L. Novotny, Phys. Rev. A 95, 061801 (2017).

[7] G. Ranjit, M. Cunningham, K. Casey, and A. A. Geraci, Phys. Rev. A 93, 053801 (2016).

[8] A. D. Rider, D. C. Moore, C. P. Blakemore, M. Louis, M. Lu, and G. Gratta, Phys. Rev. Lett. 117, 101101 (2016).

[9] Y. Arita, M. Mazilu, and K. Dholakia, Nat. Commun. 4, 2374 (2013).

[10] S. Kuhn, B. A. Stickler, A. Kosloff, F. Patolsky, K. Hornberger, M. Arndt, and J. Millen, Nat. Commun. 8, 1670 (2017).

[11] F. Monteiro, S. Ghosh, E. C. van Assendelft, and D. C. Moore, Phys. Rev. A 97, 051802 (2018).

[12] M. Schuck, D. Steinert, T. Nussbaumer, and J. W. Kolar, Sci. Adv. 4, e1701519 (2018).
[13] P. Nagornykh, J. E. Coppock, J. P. J. Murphy, and B. E. Kane, Phys. Rev. B 96, 035402 (2017).

[14] R. Zhao, A. Manjavacas, F. J. Garcia de Abajo, and J. B. Pendry, Phys. Rev. Lett. 109, 123604 (2012).

[15] Y. Guo and Z. Jacob, J. Opt. 16, 114023 (2014).

[16] A. Manjavacas, F. J. Rodríguez-Fortuño, F. J. G. de Abajo, and A. V. Zayats, Phys. Rev. Lett. 118, 133605 (2017).

[17] T. M. Hoang, Y. Ma, J. Ahn, J. Bang, F. Robicheaux, Z.-Q. Yin, and T. Li, Phys. Rev. Lett. 117, 123604 (2016).

[18] H. Shi and M. Bhattacharya, J. Mod. Opt. 60, 382 (2013).

[19] W. Lechner, S. J. M. Habraken, N. Kiesel, M. Aspelmeyer, and P. Zoller, Phys. Rev. Lett. 110, 143604 (2013).

[20] B. A. Stickler, S. Nimmrichter, L. Martinetz, S. Kuhn, M. Arndt, and K. Hornberger, Phys. Rev. A 94, 033818 (2016).

[21] H. Cavendish, Phil. Trans. R. Soc. London 88, 469 (1798).

[22] R. v. Eötvös, Math. Naturwiss. Ber. Ung. 8, 65 (1890).

[23] R. A. Beth, Phys. Rev. 50, 115 (1936).

[24] E. Adelberger, J. Gundlach, B. Heckel, S. Hoedl, and S. Schlamminger, Prog. Part. Nucl. Phys. 62, 102 (2009).

[25] Z. Xu and T. Li, Phys. Rev. A 96, 033843 (2017).

[26] V. A. Parsegian and G. H. Weiss, J. Adhes. 3, 259 (1972).

[27] Y. S. Barash, Radiophys. Quantum Electron. 21, 1138 (1978).

[28] S. J. van Enk, Phys. Rev. A 52, 2569 (1995).

[29] M. Carlesso, M. Paternostro, H. Ulbricht, and A. Bassi, arXiv:1710.08695.

[30] C. Marletto and V. Vedral, Phys. Rev. Lett. 119, 240402 (2017).

[31] S. Bose, A. Mazumdar, G. W. Morley, H. Ulbricht, M. Toroš, M. Paternostro, A. A. Geraci, P. F. Barker, M. S. Kim, and G. Milburn, Phys. Rev. Lett. 119, 240401 (2017).

[32] L. P. Neukirch, E. von Haartman, J. M. Rosenholm, and A. N. Vamivakas, Nat. Photonics 9, 653 (2015).

[33] T. M. Hoang, J. Ahn, J. Bang, and T. Li, Nat. Commun. 7, 12250 (2016).

[34] A. T. M. A. Rahman, A. C. Frangeskou, M. S. Kim, S. Bose, G. W. Morley, and P. F. Barker, Sci. Rep. 6, 21633 (2016).

[35] P. M. Johnson, C. M. van Kats, and A. van Blaaderen, Langmuir 21, 11510 (2005).

[36] See the Supplemental Material at http://link.aps.org/ supplemental/10.1103/PhysRevLett.121.033603 for more information on numerical simulations and synthesis of nanodumbbells.

[37] D. W. Mackowski, J. Aerosol Sci. 37, 242 (2006).

[38] P. Chan and B. Dahneke, J. Appl. Phys. 52, 3106 (1981).

[39] J. Corson, G. W. Mulholland, and M. R. Zachariah, Phys. Rev. E 96, 013110 (2017).

[40] J. Trojek, L. Chvátal, and P. Zemánek, J. Opt. Soc. Am. A 29, 1224 (2012).

[41] M. Pitkonen, J. Math. Phys. 47, 102901 (2006).

[42] B. T. Draine, Astrophys. J. 333, 848 (1988).

[43] K. Dholakia and P. Zemánek, Rev. Mod. Phys. 82, 1767 (2010).

[44] L. Haiberger, M. Weingran, and S. Schiller, Rev. Sci. Instrum. 78, 025101 (2007).

[45] P. H. Kim, B. D. Hauer, C. Doolin, F. Souris, and J. P. Davis, Nat. Commun. 7, 13165 (2016).

[46] G. Brambilla and D. N. Payne, Nano Lett. 9, 831 (2009).

[47] R. Reimann, M. Doderer, E. Hebestreit, R. Diehl, M. Frimmer, D. Windey, F. Tebbenjohanns, and L. Novotny, preceding Letter, Phys. Rev. Lett. 121, 033602 (2018). 